\title{
LOS TIPOS SINÓPTICOS DE JENKINSON \& COLLISON Y LA INTENSIDAD DE LA ISLA DE CALOR BARCELONESA
}

\author{
Javier MARTÍN-VIDE, M ${ }^{\mathrm{a}}$ Carmen MORENO GARCÍA, Víctor M. ARTOLA \\ y Ma José CORDOBILLA \\ Grupo de Climatología. Universidad de Barcelona. \\ jmartinvide@ub.edu, mcmoreno@ub.edu, victorm.artola@gmail.com, mj.cordobilla@gmail.com
}

\section{RESUMEN}

Las islas de calor urbanas (ICU) intensas han adquirido el carácter de riesgo durante las olas de calor en las aglomeraciones urbanas de latitudes medias, al agravar las condiciones de calor excesivo en horario nocturno, con efectos nocivos en la salud de personas mayores o enfermas. La intensidad de la ICU depende de factores geográficos, urbanos, temporales y meteorológicos. Con respecto a estos últimos, las ICU intensas se dan con viento flojo o en calma y cielos despejados o poco nubosos, condiciones que se asocian a situaciones anticiclónicas. Para verificar esta hipótesis en el caso del Área Metropolitana de Barcelona, se ha utilizado: (1) La clasificación sinóptica objetiva de Jenkinson y Collison, usando una rejilla de 16 puntos, para el período 2004-2010, y (2) las diferencias entre las temperaturas mínimas diarias de los observatorios de Barcelona (Vila Olímpica) y el aeropuerto de Barcelona de AEMET para los días del mismo período, como indicador de la intensidad de la ICU. El tipo sinóptico que presenta el mayor promedio de las diferencias es la advección del suroeste anticiclónica, con $2,7^{\circ} \mathrm{C}$. A medida que la ICU es más intensa, superior a $5,0^{\circ} \mathrm{C}$, el predominio del tipo anticiclón y de las advecciones anticiclónicas es más marcado.

Palabras clave: Área Metropolitana de Barcelona, Clasificación sinóptica de Jenkinson y Collison, isla de calor urbana, riesgo climático.

\begin{abstract}
The urban heat islands (ICUs) intense have acquired the status of risk during heat waves in urban mid-latitude agglomerations. They intensify the conditions of excessive heat at night, with harmful effects on the health of the old or sick people. The ICU intensity depends on geographical, urban, temporal and meteorological factors. With regard to the latter, the intense ICUs occur with light winds or calm, and clear skies or slightly cloudy, that are associated with anticyclonic conditions. To verify this hypothesis in the case of the Barcelona Metropolitan Area, it has been used: (1) The objective weather-types classification of Jenkinson and Collison, using a grid of 16 points, for the period 2004-2010, and (2) the differences between the minimum daily temperatures of the meteorological stations of Barcelona (Vila Olimpica) and Barcelona airport (AEMET) for the days of the same period, as an indicator of the intensity of the ICU. The synoptic type having the highest average of the differences is the anticyclonic southwest advection, with $2,7^{\circ} \mathrm{C}$. As the ICU is more intense, e.g. more than $5.0^{\circ} \mathrm{C}$, the prevalence of the anticyclone and anticyclonic advections types is starker.
\end{abstract}


Key words: Barcelona Metropolitan Area, climatic hazard, Jenkinson and Collison's weather type classification, urban heat island

\section{INTRODUCCIÓN. LA ISLA DE CALOR COMO RIESGO CLIMÁTICO POR CAUSA DEL CALENTAMIENTO GLOBAL.}

Una de las cuestiones planteadas recientemente en Climatología urbana, en el contexto del cambio climático, es el posible aumento de la intensidad de las islas de calor urbanas (ICU) por causa del calentamiento global. La cuestión tiene un gran interés: (1) Por tratarse de la interrelación entre dos escalas climáticas; (2) de cara a facilitar la detección de la señal del calentamiento global en las series térmicas de las ciudades, o, inversamente, la corrección del efecto urbano en las citadas series; y (3) por el posible agravamiento de los efectos negativos de las ICU en la salud humana durante los episodios de ola de calor.

El tercer aspecto, de producirse, reforzaría la consideración de la ICU como riesgo climático bajo ciertas condiciones meteorológicas. En los centros de las ciudades españolas del este y de la mitad sur peninsular no eran desconocidas algunas noches aisladas con temperaturas mínimas iguales o superiores a $25^{\circ} \mathrm{C}$, noches que, de momento, no han recibido una denominación "oficial" específica (a diferencia de las noches tropicales, con temperatura mínima superior a $20^{\circ} \mathrm{C}$ ). Algunas olas de calor recientes, como la de San Juan a Santiago de 2015, entre las fechas onomásticas citadas, aproximadamente, han dado lugar a varias noches seguidas con temperaturas mínimas iguales o superiores a $25^{\circ} \mathrm{C}$ en los barrios centrales de algunas ciudades, como es el caso de Barcelona. Estas condiciones térmicas nocturnas provocan un acusado estrés térmico, si no se dispone de aire acondicionado, con efectos negativos en la salud humana. Piénsese que en el interior de las viviendas, bajo esas condiciones meteorológicas, la temperatura suele ser superior. Una habitación de un piso alto mirando a poniente alcanza valores térmicos por encima de los $30^{\circ} \mathrm{C}$ durante la noche y la primera mitad de la madrugada.

Algunos investigadores han señalado que el calentamiento global no aumentará la intensidad de las ICU, es decir, la diferencia entre el centro urbano y la periferia, pero sí su gravedad en cuanto a valores nocturnos muy elevados en los barrios centrales. El hecho de que no aumente la intensidad se atribuye al desecamiento edáfico por el calentamiento de los sectores periféricos con suelo no impermeabilizado, con lo que disminuirá en ellos la pérdida de calor latente, una de las causas que realza, por contraste, la isla de calor (Hamdi et al, 2015). Suponiendo una ciudad sin variación poblacional y en la que no cambien ni los materiales de construcción, ni el SVF (factor de visión del cielo), ni la configuración de los edificios y calles, por un lado, y en la que la velocidad media del viento sea parecida y los valores de contaminación atmosférica se mantengan en niveles similares, la única causa de variación de la intensidad de la ICU es la referente a la mayor pérdida de calor latente en la periferia que en el centro de la ciudad. Sin embargo, aun sin aumentar la intensidad de la ICU, si las temperaturas globales son más elevadas, los valores térmicos en los barrios centrales de las ciudades pueden alcanzar, con el plus de la ICU, cotas peligrosas para personas mayores o con enfermedades crónicas o episódicas. Es reconocido en este sentido que la falta o la dificultad de un descanso nocturno reparador produce estrés por calor y 
debilita los organismos y los expone a las complicaciones derivadas de enfermedades de base o de la propia edad. Así, las estadísticas de mortalidad durante los episodios de ola de calor son bien concluyentes, con aumentos notables sobre el promedio de muertos según el momento del año (Gabriel y Endlicher, 2011; Lemonsu et al, 2015). Se ha venido pensando que en la mortalidad por ola de calor las temperaturas máximas eran prácticamente la única causa, cierta en el caso de los golpes de calor fatales y en otros, pero, al alimón con ellas, hay que unir el efecto, relativamente silente, pero influyente de las temperaturas mínimas elevadas, o sea, del exceso de calor nocturno. Algunos estudios hablan ya de un efecto sinérgico combinado entre la isla de calor con estos episodios de olas de calor, que agravarían las condiciones térmicas en las ciudades (Li y Bou-Zeid, 2013).

En el presente trabajo se busca confirmar la hipótesis de que las situaciones sinópticas que potencian la ICU en el Área Metropolitana de Barcelona son predominantemente anticiclónicas, en especial en el caso de las más intensas, para lo que se usa una clasificación de situaciones sinópticas objetiva. Si la intensidad de la ICU es sensible a la situación sinóptica, la predicción meteorológica se erige en una herramienta imprescindible para la alerta ante los casos en los que el citado fenómeno pueda constituir un riesgo para la población.

\section{METODOLOGÍA}

Los pasos metodológicos seguidos para este estudio han sido los siguientes:

1) Elección de la clasificación objetiva de Jenkinson y Collison (1977)(J\&C)(ver Anexo) y aplicación de la misma para clasificar sinópticamente los días del período 2004-2010, en total 2.286 fechas con datos térmicos disponibles y validados en las estaciones meteorológicas automáticas de AEMet de Barcelona (Vila Olímpica) y del aeropuerto de Barcelona.

2) Cálculo de las diferencias entre las temperaturas mínimas de las 2.286 fechas de las dos estaciones meteorológicas citadas, como indicador de la intensidad de la ICU barcelonesa (Martín-Vide et al, 2015).

3) Cálculo del promedio de las diferencias entre las temperaturas mínimas de las dos estaciones citadas para las fechas con cada uno de los 27 tipos sinópticos de $\mathrm{J} \& \mathrm{C}$.

4) Determinación de los tipos sinópticos de J\&C que suavizan y potencian la intensidad de la ICU en la AMB.

5) Cálculo porcentual de los tipos sinópticos de las fechas con una diferencia entre las temperaturas mínimas de los observatorios analizados igual o superior a $4,0^{\circ} \mathrm{C}, 5,0^{\circ} \mathrm{C}$ y $5,5^{\circ} \mathrm{C}$.

La clasificación de J\&C es una clasificación de situaciones sinópticas (weather types) objetiva o automática, cuyos tipos se obtienen siguiendo un algoritmo compuesto por unas reglas definidas, de modo que no interviene la subjetividad del operador, siendo así sus resultados reproducibles y transferibles. La clasificación de J\&C contiene 27 tipos obtenidos a partir de la presión atmosférica en superficie en un retículo de 9, 16 o más puntos que englobe el área objeto de estudio. En nuestro caso, se ha usado el retículo octogonal de 16 puntos con puntos extremos en los paralelos $30^{\circ} \mathrm{N}$ y $50^{\circ} \mathrm{N}$ y en los meridianos $20^{\circ} \mathrm{W}$ y $10^{\circ} \mathrm{E}$ utilizado en Meseguer (2015)(figura 1). 


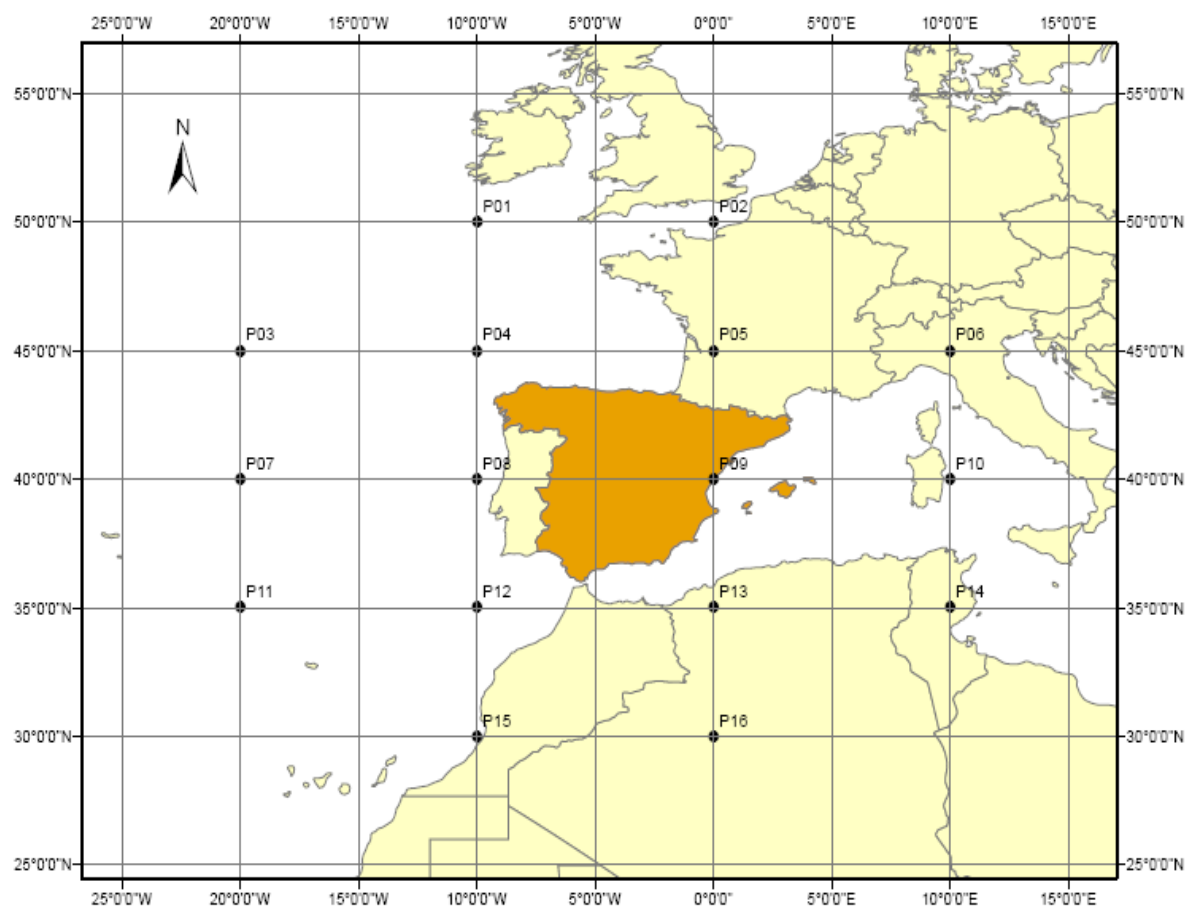

Figura 1: Retículo octogonal de 16 puntos usado para la clasificación sinóptica de Jenkinson y Collison. Fuente: Meseguer (2015).

La descripción básica de los 27 tipos de J\&C se resume en la tabla 1. Los correspondientes modelos isobáricos se representan en la figura 2.

N, NE, E, SE, S, SW, W y NW: Advecciones puras del N, NE, E, SE, S, SW, W y NW, respectivamente, constituidas por un haz de isobaras dispuestas rectilíneamente sobre la Península Ibérica marcando flujos de viento de las citadas direcciones.

AN, ANE, AE, ASE, AS, ASW, AW y ANW: Advecciones mixtas de carácter anticiclónico del N, NE, E, SE, S, SW, W y NW, respectivamente, constituidas por isobaras con curvatura anticiclónica sobre la Península Ibérica marcando flujos de viento de las citadas direcciones.

CN, CNE, CE, CSE, CS, CSW, CW y CNW: Advecciones mixtas de carácter ciclónico del N, NE, E, SE, S, SW, W y NW, respectivamente, constituidas por isobaras con curvatura ciclónica sobre la Península Ibérica marcando flujos de viento de las citadas direcciones.

A: Anticiclón centrado sobre la Península Ibérica.

C: Depresión o borrasca centrada sobre la Península Ibérica.

U: Campo de presión en superficie poco definido, sin apenas gradiente bárico, caracterizado por la ausencia de isobaras o la presencia de algunas laxas (pantano barométrico).

Tabla 1: Descripción básica de los tipos de Jenkinson y Collison. Fuente: Elaboración propia. 

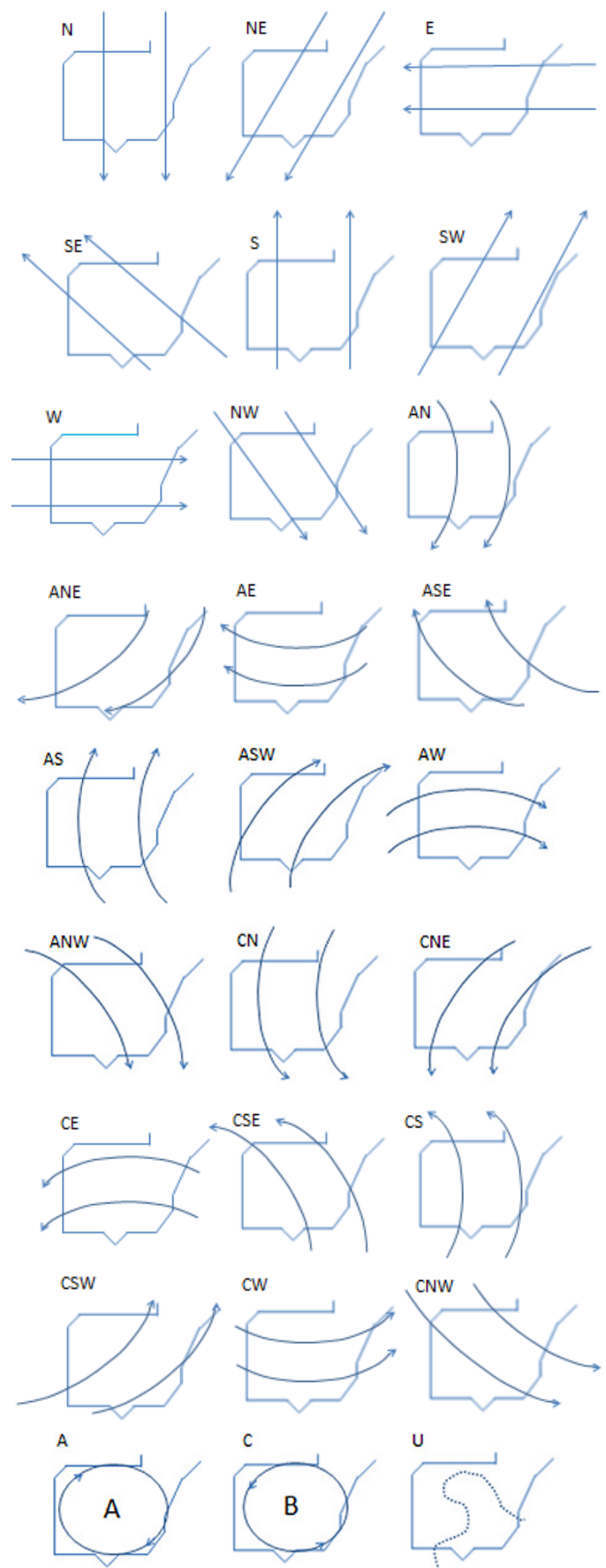

Figura 2: Configuración isobárica básica de los 27 tipos sinópticos de Jenkinson y Collison sobre la Península Ibérica. (Las flechas representan isobaras). Fuente: Elaboración propia. 


\section{RESULTADOS}

En la tabla 2 se presentan el número de casos por cada tipo sinóptico de J\&C, el porcentaje sobre el total y la diferencia media entre las temperaturas mínimas de los observatorios de Barcelona Vila Olímpica y el aeropuerto de Barcelona de los 2.286 días analizados. Solo tres tipos engloban más del 40\% del total de días: El A (19,4\%), el C (12,3\%) y el E (10,9\%). Excepto el tipo CS, el menos frecuente, todos los demás contienen al menos 20 casos.

\begin{tabular}{|c|c|c|c|}
\hline Tipo de Jenkinson y Collison & $N^{o}$ & $\%$ & $\begin{array}{c}\text { Media Dif.Mínimas } \\
\text { BCN-V.Olimp.y el aerop. }\end{array}$ \\
\hline N (Advectivo del N) & 84 & 3,7 & 1,6 \\
\hline NE (Advectivo del NE) & 97 & 4,2 & 1,4 \\
\hline E (Advectivo del E) & 249 & 10,9 & 1,5 \\
\hline SE (Advectivo del SE) & 120 & 5,2 & 2,0 \\
\hline S (Advectivo del S) & 45 & 2,0 & 1,9 \\
\hline SW (Advectivo del SW) & 81 & 3,5 & 2,1 \\
\hline W (Advectivo del W) & 98 & 4,3 & 2,2 \\
\hline NW (Advectivo del NW) & 95 & 4,2 & 1,7 \\
\hline AN (Advect. del N anticiclónico) & 23 & 1,0 & 1,7 \\
\hline ANE(Advect del NE anticiclónico) & 28 & 1,2 & 2,1 \\
\hline AE (Advect. del E anticiclónico) & 61 & 2,7 & 2,2 \\
\hline ASE(Advect. del SE anticiclónico) & 37 & 1,6 & 2,2 \\
\hline AS (Advect. del S anticiclónico) & 23 & 1,0 & 2,2 \\
\hline ASW(Advec.del SW anticiclónico) & 21 & 0,9 & 2,7 \\
\hline AW (Advect. del W anticiclónico) & 29 & 1,3 & 2,0 \\
\hline ANW(Advec.del NWanticiclónico) & 35 & 1,5 & 2,2 \\
\hline CN (Advect. del N ciclónico) & 28 & 1,2 & 1,1 \\
\hline CNE (Advect. del NE ciclónico) & 42 & 1,8 & 1,0 \\
\hline CE (Advect. del E ciclónico) & 57 & 2,5 & 1,3 \\
\hline CSE (Advect. del SE ciclónico) & 27 & 1,2 & 1,5 \\
\hline CS (Advect. del S ciclónico) & 9 & 0,4 & 2,3 \\
\hline CSW (Advect. del SW ciclónico) & 20 & 0,9 & 1,9 \\
\hline CW (Advect. del W ciclónico) & 38 & 1,7 & 2,5 \\
\hline CNW (Advect. del NW ciclónico) & 38 & 1,7 & 1,5 \\
\hline A (Anticiclón) & 444 & 19,4 & 1,9 \\
\hline C (Depresión) & 282 & 12,3 & 1,3 \\
\hline U (Indeterminado) & 175 & 7,7 & 1,3 \\
\hline Total & 2.286 & 100 & \\
\hline
\end{tabular}

Tabla 2: Diferencia media entre las temperaturas mínimas de Barcelona-Vila Olímpica y el aeropuerto de Barcelona según los tipos sinópticos de Jenkinson y Collison, en el período 2004-2010. Fuente: Elaboración propia. 
A la vista de la tabla 2, y considerando como tipos sinópticos que potencian la ICU en el Área Metropolitana de Barcelona los de promedios de las diferencias entre las temperaturas mínimas superiores a $2,0^{\circ} \mathrm{C}$ (valor en negrita en la tabla 2 ) y como tipos sinópticos que la suavizan, los inferiores a $1,5^{\circ} \mathrm{C}$ (valor en cursiva en la tabla 2 ), los principales resultados son:

1) La intensidad de la isla de calor del Área Metropolitana de Barcelona es sensible a la situación sinóptica, por lo que la predicción meteorológica es de gran importancia en la previsión de la intensidad de la misma en cada noche, así como en el establecimiento de alertas a la población en los casos críticos.

2) Todos los tipos sinópticos dan diferencias térmicas medias positivas entre las temperaturas mínimas de la ciudad y su periferia, lo que refleja la alta frecuencia y la notable intensidad de la ICU barcelonesa.

3) Los valores extremos de las diferencias térmicas medias van desde $1,0^{\circ} \mathrm{C}$ para la advección del nordeste ciclónica $(\mathrm{CNE})$ hasta $2,7^{\circ} \mathrm{C}$ para la advección del suroeste anticiclónica (ASW), lo que supone un rango apreciable teniendo en cuenta que se trata de promedios.

4) Los tipos sinópticos que potencian la ICU son las advecciones del tercer cuadrante (SW y W) y los anticiclónicos mixtos (ANE, AE, ASE, AS, ASW y ANW), estos últimos asociados, casi siempre, a condiciones de estabilidad atmosférica, así como dos ciclónicos mixtos (CW y CS, este último con una representación residual). La presencia de tipos advectivos (mixtos) entre los que producen intensidades más altas ya fue señalada por Moreno-García (1994). El tipo A, o anticiclón puro, no aparece entre las situaciones sinópticas que potencian la ICU, presumiblemente por su alta frecuencia en la clasificación usada.

5) Los tipos sinópticos que producen islas de calor débiles o impiden el fenómeno son el tipo $\mathrm{C}$, o ciclónico puro, el indeterminado (U), el advectivo del nordeste (NE) y los ciclónicos mixtos del primer cuadrante (CN, CNE y CE), la mayoría de ellos asociados a condiciones de inestabilidad atmosférica o atmósferas con estratificación neutra.

Con el objeto de indagar más en la hipótesis de partida se ha buscado qué tipos sinópticos son los de los días con las ICU más intensas. Para ello se identificaron las fechas con una diferencia entre las temperaturas mínimas de los dos observatorios empleados igual o superior a $4,0^{\circ} \mathrm{C}, 5,0^{\circ} \mathrm{C}$ y $5,5^{\circ} \mathrm{C}$. Los 134 casos con una diferencia igual o superior a $4,0^{\circ} \mathrm{C}$ se distribuyeron así: $59(44,0 \%)$ con los tipos A o advectivos anticiclónicos; 50 (37,3\%) advectivos y $25(18,7 \%)$ con los tipos C o advectivos ciclónicos. Los 29 casos con una diferencia igual o superior a $5,0^{\circ} \mathrm{C}$ presentaron la siguiente distribución: 14 (48,3\%) con los tipos A o advectivos anticiclónicos; 8 (27,6\%) advectivos y $7(24,1 \%)$ con los tipos $\mathrm{C}$ o advectivos ciclónicos. Y los 7 casos con una diferencia igual o superior a $5,5^{\circ} \mathrm{C}$ ocurrieron con los tipos A o advectivos anticiclónicos en 6 casos $(85,7 \%)$ y solo en uno con el tipo C. En resumen, a medida que la isla de calor es más intensa el predominio de los tipos sinópticos $\mathrm{A}$ y advectivos anticiclónicos es más predominante. Esta agrupación del tipo A y de los advectivos anticiclónicos está presente en 701 días en el período analizado, es decir, en un 30,7\% del total de días, porcentaje significativamente inferior al de los casos con diferencias iguales o superiores a los tres umbrales establecidos para señalar las fechas con ICU intensas. Se concluye: 
6) Cuanto más intensa es la isla de calor más presencia tienen los tipos A y advectivos anticiclónicos; por encima de $5,0^{\circ} \mathrm{C}$ están presentes en más del $50 \%$ de los casos.

\section{CONCLUSIONES}

Se ha confirmado la hipótesis de partida de que las situaciones sinópticas que potencian la ICU en el Área Metropolitana de Barcelona son predominantemente de carácter anticiclónico, en especial en el caso de las más intensas, sea el tipo anticiclón o las advecciones anticiclónicas.

La situación sinóptica con una mayor diferencia térmica media entre la ciudad y el aeropuerto es la advección del suroeste anticiclónica (ASW), con 2,7 $7^{\circ}$.

La predicción meteorológica ha de contemplarse como una herramienta imprescindible para la alerta, en el Área Metropolitana de Barcelona, ante los casos de ICU intensas en verano, que, en el contexto del cambio climático, pueden constituir un riesgo para la población.

\section{AGRADECIMIENTOS}

La presente investigación se ha desarrollado en el marco del Observatorio Metropolitano del Cambio Climático (METROBS) del Área Metropolitana de Barcelona, en concreto se vincula al proyecto "La isla de calor en el Área Metropolitana de Barcelona y la adaptación al cambio climático", dirigido por el primer autor. Asimismo, se inserta en los trabajos del Grupo de Climatología (2014SGR300) de la Generalitat de Catalunya y emplea metodologías desarrolladas en el proyecto CSO2014-55799. C2-1-R del Ministerio de Economía y Competitividad. Gracias especiales a Óliver Meseguer por la base de datos de Jenkinson y Collison.

\section{REFERENCIAS}

Gabriel, K.M. y Endlicher, W.R. (2011): Urban and rural mortality rates during heat waves in Berlin and Brandenburg, Germany. Environmental Pollution, 159(8-9), 2044-2050. doi: 10.1016/j.envpol.2011.01.016

Grimalt, M.; Tomàs, M.; Alomar, G.; Martín-Vide, J. y Moreno-García, M. C. (2013): Determination of the Jenkinson and Collison's weather types for the western Mediterranean basin over the 1948-2009 period. Temporal analysis. Atmósfera, 26(1), 75-94. doi: 10.1016/S0187-6236(13)71063-4

Hamdi, R.; Giot, O.; De Troch, R.; Deckmyn, A. y Termonia, P. (2015): Future climate of Brussels and Paris for the 2050s under the A1B scenario, Urban Climate, 12, 160-182. doi:10.1016/j.uclim.2015.03.003

Lemonsu, A.; Viguié, V.; Daniel, M. y Masson, V. (2015): Vulnerability to heat waves: Impact of urban expansion scenarios on urban heat island and heat stress in Paris (France). Urban Climate, 14(4), 586-605. doi:10.1016/j.uclim.2015.10.007

Li, D. y Bou-Zeid, E. (2013): Synergistic interactions between urban heat islands and heat waves: the impact in cities is larger than the sum of its parts. Journal of Applied Meteorology and Climatology, 52, 2051-2064. doi: 10.1175/JAMC-D-13-02.1 
Martín-Vide, J.; Sarricolea, P. y Moreno-García, M.C. (2015): On the definition of urban heat island intensity: the "rural" reference. Front. Earth Sc., 3:24. doi: $10.3389 /$ feart.2015.00024

Meseguer, Ó. (2015): La fractalidad temporal de la precipitación en la España Peninsular y Baleares, Tesis doctoral, Universidad de Barcelona.

Moreno-García, M.C.(1994): Intensity and form of the urban heat island in Barcelona. Int. Jour. of Climatology, 14, 705-710. doi:10.1002/joc.33701.40609

\section{ANEXO: La clasificación objetiva de Jenkinson y Collison}

La clasificación de Jenkinson y Collison se basa en 7 variables deducidas exclusivamente a partir de la presión atmosférica en superficie. Estas variables son:

W, componente zonal del viento (superficial) geostrófico, calculada, por ejemplo, como gradiente de presión, por ejemplo, entre $35^{\circ}$ y $45^{\circ} \mathrm{N}$ para la Península Ibérica;

$\mathrm{S}$, componente meridiana del viento (superficial) geostrófico, calculada, por ejemplo, como gradiente de presión, por ejemplo, entre $0^{\circ}$ y $10^{\circ} \mathrm{W}$ para la Península Ibérica;

$\mathrm{D}$, dirección del viento ( ${ }^{\circ}$ azimut);

$\mathrm{F}$, velocidad del viento $(\mathrm{m} / \mathrm{s})$;

ZW , componente zonal de la vorticidad;

ZS , componente meridiana de la vorticidad;

Z, vorticidad total.

Para el ámbito de la Península Ibérica puede usarse el retículo octogonal de 16 puntos, P01, P02, $\mathrm{P} 03, \ldots, \mathrm{P} 16$ de la figura 1 , cuyos puntos son intersecciones de los paralelos $30^{\circ}, 35^{\circ}, 40^{\circ}, 45^{\circ}$ y $50^{\circ} \mathrm{N}$ con los meridianos $10^{\circ} \mathrm{E}, 0^{\circ}, 10^{\circ} \mathrm{W}$ y $20^{\circ} \mathrm{W}$, exceptuando los vértices del retículo. Podrían usarse otros retículos (Grimalt et al, 2013). Conociendo la presión atmosférica en superficie en los 16 puntos, sean, usando la misma notación, P01, P02, P03,..,P16, se hallan los valores de las 7 variables. Las expresiones analíticas empleadas para su cálculo son las siguientes (Meseguer, 2015):

$\mathrm{W}=0,5(\mathrm{P} 12+\mathrm{P} 13)-0,5(\mathrm{P} 4+\mathrm{P} 5)$

$\mathrm{S}=1,3052[0,25(\mathrm{P} 5+2 \mathrm{P} 9+\mathrm{P} 13)-0,25(\mathrm{P} 4+2 \mathrm{P} 8+\mathrm{P} 12)]$

$\mathrm{D}=\operatorname{arctg}(\mathrm{W} / \mathrm{S})$

$\mathrm{F}=\left(\mathrm{W}^{2}+\mathrm{S}^{2}\right)^{1 / 2}$

$\mathrm{ZW}=1,1207[0,5(\mathrm{P} 15+\mathrm{P} 16)-0,5(\mathrm{P} 8+\mathrm{P} 9)]-0,909[0,5(\mathrm{P} 8+\mathrm{P} 9)-0,5(\mathrm{P} 1+\mathrm{P} 2)]$

$\mathrm{ZS}=0,852[0,25(\mathrm{P} 6+2 \mathrm{P} 10+\mathrm{P} 14)-0,25(\mathrm{P} 5+2 \mathrm{P} 9+\mathrm{P} 13)-0,25(\mathrm{P} 4+2 \mathrm{P} 8+\mathrm{P} 12)+0,25(\mathrm{P} 3+2 \mathrm{P} 7+\mathrm{P} 11)]$

$\mathrm{Z}=\mathrm{ZW}+\mathrm{ZS}$

A partir de los valores de estas 7 variables se aplican las 5 reglas del método de Jenkinson y Collison siguientes:

1) La dirección del flujo viene dada por D (se usa una rosa de vientos de 8 rumbos y se tiene en cuenta el signo de $\mathrm{W}$ y de $\mathrm{S}$ ).

2) $\operatorname{Si}|\mathrm{Z}|<\mathrm{F}$, existe un tipo advectivo o direccional puro, definido según la regla 1 (N, NE, E, SE, S, SW, W y NW).

3) $\mathrm{Si}|\mathrm{Z}|>2 \mathrm{~F}$, existe un tipo ciclónico (C), si $\mathrm{Z}>0$, o anticiclónico (A), si $Z<0$.

4) $\operatorname{Si~} \mathrm{F}<|\mathrm{Z}|<2 \mathrm{~F}$, existe un tipo híbrido, según el signo de $\mathrm{Z}$ (regla 3) y la dirección del flujo (regla 1) (CN, CNE, CE, CSE, CS, CSW, CW, CNW, AN, ANE, AE, ASE, AS, ASW, AW y ANW).

5) Si $F<4,8$ y $|Z|<4,2$, existe un tipo indeterminado (U).

De este modo se obtienen los 27 tipos.

(Hay que indicar que las constantes de la regla 5 pueden variar dependiendo del realce que quiera darse al tipo indeterminado) 